Z. Phys. Chem. 216 (2002) 521-533

(C) by Oldenbourg Wissenschaftsverlag, München

\title{
Complex Pacemakers and Wave Sinks in Heterogeneous Oscillatory Chemical Systems
}

\author{
By Michael Stich* and Alexander S. Mikhailov \\ Abteilung Physikalische Chemie, Fritz-Haber-Institut der Max-Planck-Gesellschaft, \\ Faradayweg 4-6, D-14195 Berlin, Germany
}

Dedicated to Prof. Dr. Friedemann W. Schneider

(Received August 10, 2001; accepted October 15, 2001)

\section{Reaction-Diffusion Systems / Pattern Formation / Eckhaus Instability / Wave Dispersion}

We investigate pattern formation in oscillatory reaction-diffusion systems where wave sources and sinks are created by a local shift of the oscillation frequency. General properties of resulting wave patterns in media with positive and negative dispersion are discussed. It is shown that phase slips in the wave patterns develop for strong frequency shifts, indicating the onset of desynchronization in the medium.

\section{Introduction}

Nonequilibrium chemical reactions can display a rich variety of complex kinetic regimes, including oscillations, wave propagation, and formation of regular or chaotic spatiotemporal patterns $[1,2]$. The first wave pattern discovered in the Belousov-Zhabotinsky reaction was the target pattern [3]. It consisted of concentric waves that were periodically emitted from a small central region. Since then, similar spatiotemporal structures have also been observed in other chemical, physical, and biological systems [4-8].

Target patterns may already be found in uniform systems, as a result of nonlinear kinetics and diffusion. Such self-organized stable target patterns have been described in several models (e.g., [9-11]). There is also experimental evidence that some target patterns in the Belousov-Zhabotinsky (BZ) reaction are not related to any heterogeneity and may be autonomous [12]. Nonetheless, the great majority of target patterns observed in chemical systems are associated with the presence of a local heterogeneity (a dust particle, gas bubble,

* Corresponding author. E-mail: stich@fhi-berlin.mpg.de 
etc.) that modifies locally the properties of the medium and gives rise to a pacemaker. Indeed, by careful filtering of the BZ solution, the number of evolving target patterns can be reduced. The developing patterns have a range of operation frequencies, indicating that the frequency is not uniquely determined by the medium parameters. Furthermore, in the experiment described in [13] the activity of a pacemaker, generating a target pattern in the BZ reaction, was suppressed by application of another, high-frequency wave source. When this other source was however removed, the initial pacemaker reappeared at the same location with the same frequency.

The properties of heterogeneous pacemakers and their target wave patterns have been analyzed in a number of theoretical studies [14-18]. Creation of pacemakers through application of noise to a photosensitive BZ medium has been recently reported [19]. Nonetheless, such spatiotemporal patterns have so far received much less attention than, for example, rotating spiral waves which represent another kind of pattern in oscillatory media. In this paper we present a brief review of the theory of heterogeneous pacemakers and wave sinks, and discuss their instabilities and complex dynamics that may be experimentally observed. Particularily, experimental studies with the photosensitive BZ reaction, where controlled heterogeneities may easily be created by application of appropriate illumination patterns, would be of much interest here.

As a theoretical model to study these effects, we choose the complex Ginzburg-Landau equation (CGLE) [20]. Though this equation is strictly applicable only close to a soft onset of oscillations where oscillations have small amplitudes and are approximately harmonical, its predictions are known to be qualitatively valid in a broader neighbourhood of the supercritical Hopf bifurcation. It can be shown that stable self-organized pacemakers are not possible in the CGLE [18]. If such a wave source is initially formed by preparing special initial conditions, phase diffusion leads to spreading of the central core region, the increase of the wavelength, and the final disappearance of the target pattern, which transforms into uniform oscillations.

Hence, to create a pacemaker in the CGLE, the medium should be nonuniform, possessing a small localized region with modified parameters. Then, the local oscillation frequency inside this region is different from outside and this region may form a pacemaker which generates a spatially extended wave pattern. Such pacemakers and their wave patterns are in the focus of this article. Additionally, localized wave patterns representing sinks will also be considered.

We show that, as the difference between the local oscillation frequency in the pacemaker region and the frequency of bulk oscillations is increased, the oscillations inside of the pacemaker may desynchronize with those present outside of the core. The pacemaker is then too fast to entrain the rest of the system by emission of waves and the resulting spatiotemporal wave pattern is governed by the repeated appearance of phase slips. This dynamical behavior is closely related to the Eckhaus instability of traveling waves. 
This article is organized as follows. First, the complex Ginzburg-Landau equation is introduced (Sect. 2) and the phase dynamics approximation for pacemakers is discussed (Sect. 3). In the same section, basic properties of weak pacemakers and sinks and their wave patterns in the one-dimensional CGLE are summarized. In Sect. 4, we investigate the properties of strong pacemakers and sinks whose operation is accompanied by repeated phase slips. The paper ends with a discussion of the obtained results.

\section{The complex Ginzburg-Landau equation}

Reaction-diffusion systems can display different types of oscillatory dynamics. However, in the vicinity of a supercritical Hopf bifurcation, where oscillations have small amplitude and are approximately harmonical, all such systems are described by the same model - the complex Ginzburg-Landau equation (CGLE). The predictions based on this universal amplitude equation often remain qualitatively correct even further away from the bifurcation point, where the oscillation amplitudes are moderate and deviations from harmonicity are significant. The derivation of a CGLE for a specific reaction-diffusion model is explained in $[18,21]$. The equation reads

$$
\partial_{t} A=(1-\mathrm{i} \omega) A-(1+\mathrm{i} \alpha)|A|^{2} A+(1+\mathrm{i} \beta) \nabla^{2} A,
$$

where $A$ is the complex oscillation amplitude, $\omega$ is the basic frequency of the system near to the bifurcation point, $\alpha$ is the nonlinear frequency shift, and $\beta$ is the linear dispersion coefficient. The frequency of stable uniform oscillations $A(t)=\exp (-\mathrm{i} \Omega t)$ in this system is $\Omega=\omega+\alpha$ and their amplitude is $|A|=1$. Eq. (1) is written in the dimensionless form where time is measured in units which are inversely proportional to the distance from the Hopf bifurcation point. Therefore, close to the Hopf bifurcation the condition $\omega \gg 1$ is always satisfied. The parameters $\alpha$ and $\beta$ are generally of order unity. Note that by going to a co-rotating coordinate frame, $\omega$ can be scaled out of the CGLE.

The CGLE has plane wave solutions $A(x, t)=\sqrt{1-k^{2}} \exp \left(\mathrm{i} k x-\mathrm{i} \omega_{k} t\right)$ [20]. The dependence of the wave frequency $\omega_{k}$ on the wavenumber $k$ constitutes the dispersion relation $\omega_{k}$. It is given by

$$
\omega_{k}=\Omega+(\beta-\alpha) k^{2}
$$

When $\beta-\alpha>0$, the frequency increases with the wavenumber and the waves have positive dispersion. In the opposite case, the dispersion is negative. The phase velocity is defined as $v_{\mathrm{p}}=\omega_{k} / k$ and given by

$$
v_{\mathrm{p}}=\Omega / k+(\beta-\alpha) k .
$$


It characterizes the speed at which the positions of wave maxima move. The group velocity is defined as $v_{\mathrm{g}}=\partial \omega_{k} / \partial k$, so that

$$
v_{\mathrm{g}}=2(\beta-\alpha) k .
$$

It determines the velocity at which small perturbations (wave packages) propagate through the medium.

Suppose that the oscillation frequency $\omega$ is changed by an amount $\Delta \omega$ within a small region of radius $R$, so that

$$
\omega(x)=\left\{\begin{array}{lll}
\omega & \text { for } & |x|>R \\
\omega+\Delta \omega & \text { for } & |x| \leq R .
\end{array}\right.
$$

Then, the region with the modified local oscillation frequency may become either a source or a sink of traveling waves.

\section{Pacemakers and sinks in the phase dynamics approximation}

Wave patterns can be described using the phase dynamics approximation if the wavelength is large and - more generally, phase perturbations are smooth [18]. Introducing the phase $\phi$ and the (real) amplitude $\rho$ as $A=\rho \exp (-\mathrm{i} \phi)$ and substituting this into the CGLE, we obtain the two equations

$$
\begin{aligned}
& \partial_{t} \rho=\left(1-\rho^{2}\right) \rho+\nabla^{2} \rho-\rho(\nabla \phi)^{2}+\beta \rho \nabla^{2} \phi+2 \beta \nabla \phi \nabla \rho, \\
& \partial_{t} \phi=\omega(x)+\alpha \rho^{2}+(2 / \rho) \nabla \rho \nabla \phi+\nabla^{2} \phi-(\beta / \rho) \nabla^{2} \rho+\beta(\nabla \phi)^{2} .
\end{aligned}
$$

For smooth phase perturbations with characteristic lengths $L \gg 1$, the local amplitude $\rho$ of oscillations follows adiabatically the dynamics of the phase, so that $\rho^{2} \approx 1-(\nabla \phi)^{2}+\beta \nabla^{2} \phi$. There, we have omitted terms of higher order than $L^{-2}$. Consequently, Eqs. (6) are reduced to a single dynamical equation for the local oscillation phase,

$$
\partial_{t} \phi=\omega(x)+\alpha+(\beta-\alpha)(\nabla \phi)^{2}+(1+\alpha \beta) \nabla^{2} \phi .
$$

In this paper we assume that the Benjamin-Feir-Newell condition $1+\alpha \beta>0$ is satisfied and uniform oscillations are modulationally stable.

After applying the nonlinear Hopf-Cole transformation

$$
\phi=\frac{1+\alpha \beta}{\beta-\alpha} \ln Q
$$

to the phase equation (7), the following linear equation for new variable $Q$ is yielded:

$$
\partial_{t} Q=\frac{\beta-\alpha}{1+\alpha \beta}(\omega(x)+\alpha) Q+(1+\alpha \beta) \nabla^{2} Q .
$$


A general solution of this equation is (see also [18])

$$
Q(x, t)=\sum_{n} C_{n} \exp \left(\lambda_{n} t\right) Q_{n}(x),
$$

where $\lambda_{n}$ and $Q_{n}(x)$ are the eigenvalues and eigenfunctions of the linear differential operator

$$
\widehat{L}=(1+\alpha \beta) \nabla^{2}+\frac{\beta-\alpha}{1+\alpha \beta}(\omega(x)+\alpha) .
$$

Note that all eigenvalues of this operator are real. Generally, the operator will have both a discrete and a continuous spectrum. For long times, the solution with the largest eigenvalue $\lambda_{0}$ will dominate the expansion. In this case we have $Q(x, t) \simeq C_{0} \exp \left(\lambda_{0} t\right) Q_{0}(x)$ and

$$
\phi(x, t) \simeq \frac{1+\alpha \beta}{\beta-\alpha}\left(\lambda_{0} t+\ln Q_{0}(x)\right) .
$$

If the largest eigenvalue $\lambda_{0}$ belongs to the discrete spectrum of operator $\widehat{L}$, the corresponding eigenfunction is localized and $Q_{0}(x) \propto \exp \left(-\kappa_{0}|x|\right)$ for $|x| \rightarrow \infty$, where $\kappa_{0}=\sqrt{\lambda_{0} /(1+\alpha \beta)}$. Hence, far from the center we have

$$
\phi(x, t) \simeq \frac{1+\alpha \beta}{\beta-\alpha}\left(\lambda_{0} t-\kappa_{0}|x|\right) .
$$

This means that far from the central region, where the local oscillation frequency is modified, propagation of approximately plane waves takes place. Note that $\kappa_{0}$ is related to the wavenumber $k_{0}$ of these waves through $k_{0}=$ $\kappa_{0}(1+\alpha \beta) /(\beta-\alpha)$.

In contrast to this, if the operator $\widehat{L}$ has no discrete spectrum, its largest eigenvalue belongs to the continuum spectrum and the respective eigenfunction has asymptotics $Q_{0}(x) \rightarrow$ const at $|x| \rightarrow \infty$. Therefore,

$$
\phi(x, t) \simeq \frac{1+\alpha \beta}{\beta-\alpha}\left(\lambda_{0} t+\text { const }\right)
$$

far from the central region and uniform oscillations are taking place there.

Whether the largest eigenvalue of the operator $\widehat{L}$ belongs to the discrete or continuous spectrum, is determined by the dispersion coefficient $\beta-\alpha$ and the frequency shift $\Delta \omega$. For $(\beta-\alpha) \Delta \omega>0$ the largest eigenvalue belongs to the discrete spectrum, for $(\beta-\alpha) \Delta \omega<0$ to the continuous spectrum. Below we apply these results (see also [22]) to discuss properties of pacemakers (sources) and sinks in media with different dispersion and show examples of resulting wave patterns. 


\section{A. Positive dispersion $(\beta>\alpha)$}

When the local oscillation frequency is increased $(\Delta \omega>0)$ inside a core region of radius $R$, this region becomes a source of waves and a classical target pattern of outwards propagating waves is formed. In one-dimensional media, the wavenumber $k_{0}$ of generated waves is determined by the equation

$$
R=\frac{1+\alpha \beta}{(\beta-\alpha) \sqrt{k_{\max }^{2}-k_{0}^{2}}} \tan ^{-1}\left(\frac{k_{0}}{\sqrt{k_{\max }^{2}-k_{0}^{2}}}\right)
$$

where $k_{\max }=\kappa_{\max }(1+\alpha \beta) /(\beta-\alpha)=\sqrt{\Delta \omega /(\beta-\alpha)}$ is the maximum wavenumber reached for very large cores. This equation is found by matching the solution valid far from the core [Eq. (13)] with the solution inside the core where $Q_{0}(x) \propto \cos \left(\sqrt{\kappa_{\max }^{2}-\kappa_{0}^{2}}|x|\right)$. For a fixed frequency shift $\Delta \omega$, the wavenumber increases monotonously with the radius $R$. The pacemaker frequency is

$$
\Omega_{0}=\Omega+(\beta-\alpha) k_{0}^{2} .
$$

As a result of wave propagation, the oscillation frequency becomes the same throughout the system. An example of this target wave pattern is shown in Fig. 1a. Waves leave the core region and propagate outwards, showing that the

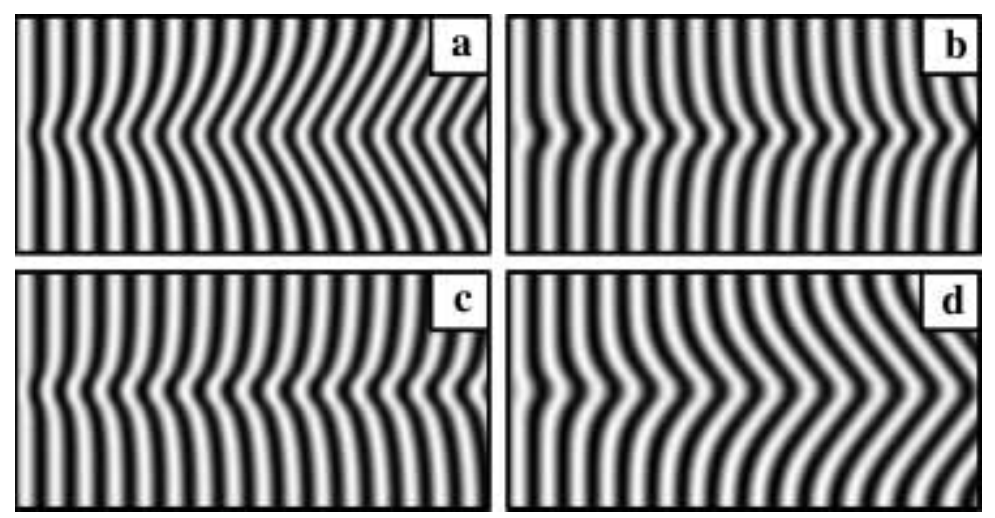

Fig. 1. Space-time diagrams of wave patterns. (a) Wave source in a medium with positive dispersion (target pattern); $\Delta \omega=0.2, \alpha=0.5, \beta=1.0$; (b) wave sink in a medium with positive dispersion; $\Delta \omega=-0.2, \alpha=0.5, \beta=1.0$; (c) wave sink in a medium with negative dispersion; $\Delta \omega=0.2, \alpha=-0.5, \beta=-1.0$; (d) wave source in a medium with negative dispersion (inward propagating target pattern); $\Delta \omega=-0.2, \alpha=-0.5, \beta=-1.0$. Other parameters are $\Omega=0.5$ and $R=4$; the displayed time interval is $T=200$ and the system size is $L=80$. Space is displayed along the vertical axis, time runs along the horizontal axis, and $\operatorname{Re} A$ is displayed in grey scale. In all simulations no-flux boundary conditions are used. 
phase velocity is directed outwards. Since the waves are able to entrain the whole system, the core region represents indeed a wave source and the group velocity of the waves is hence directed outwards.

When the local oscillation frequency is decreased $(\Delta \omega<0)$ inside the core region, a localized wave sink pattern is produced. Waves initiate near the core boundary and propagate inwards (see Fig. 1b). Now, both the group and the phase velocities are directed towards the center. As a result, the frequency of the core oscillations is increased to the value of uniform oscillations $\Omega$, giving rise to a pattern where a constant phase shift between the wave sink and rest of the medium is present. Also in this case, the oscillation frequency is constant throughout the system.

\section{B. Negative dispersion $(\beta<\alpha)$}

When the local oscillation frequency is increased $(\Delta \omega>0)$ inside a core of radius $R$, such a heterogeneity leads to a localized wave pattern with frequency $\Omega$. An example of this pattern is shown in Fig. 1c. Waves are initiated in the core and propagate outwards, but then die at the core boundary. Since dispersion is negative, the group velocity has here the opposite sign with respect to the phase velocity. Hence, the group velocity is directed inwards for this wave pattern and it actually represents a sink of waves.

If the local oscillation frequency is decreased $(\Delta \omega<0)$ inside the core region, an extended wave pattern is formed. Outside of the core of radius $R$, the medium is filled with propagating waves. The wavenumber $k_{0}$ of these waves is again determined by Eq. (15) where, however, $(\beta-\alpha)$ is replaced by $(\alpha-\beta)$. The frequency $\Omega_{0}$ of generated waves is given by Eq. (16). Note that it is lower than the frequency $\Omega$ of uniform oscillations. The waves in this pattern propagate inwards (Fig. 1d) since the direction of the phase velocity is reversed with respect to the group velocity which is directed outwards. Despite the fact that waves are moving inwards, this pattern represents a wave source and thus a pacemaker.

Though theoretical studies of wave patterns in oscillatory media with negative dispersion have been performed (e.g., [23]), this kinetic regime remains poorly explored and no experimental data on the properties of pacemakers in such systems seem to be available.

Note that to simplify the visualization in the above examples, we have chosen a relatively low frequency $\Omega=0.5$ of uniform oscillations. Similar patterns are observed for high oscillation frequencies.

Fig. 2 displays spatial distributions of the local wavenumber, defined as $k=-\nabla \phi$, for the four different kinds of wave patterns shown in Fig. 1. For the sources ( $c f$. Fig. 1a,d) the wavenumber becomes constant outside the core (but then goes to zero at the no-flux boundary). The different signs of $k$ reflect the different directions of the group velocities. In contrast to this, the local wavenumber rapidly falls down to zero in the case of sinks ( $c f$. Fig. 1b,c), showing that no plane waves are present. 

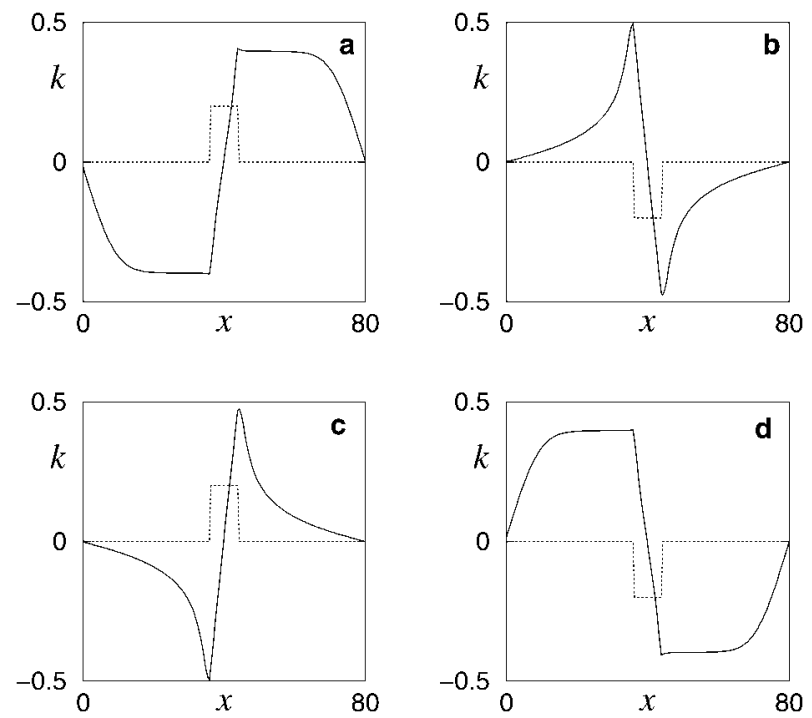

Fig. 2. Spatial distributions of the local wavenumber $k$ at fixed time moments for the patterns displayed in Fig. 1(a,b,c,d). The local frequency shift $\Delta \omega$ is displayed as a dotted line.

The phase dynamics approximation is valid only when phase gradients (i.e., local wavenumbers) remain sufficiently small. Therefore, the above theoretical results based on the phase dynamics approximation hold only for weak pacemakers and sinks, which are found when the frequency difference $\Delta \omega$ in the core region is not too large. The properties of strong pacemakers and sinks with large frequency difference $\Delta \omega$ are considered in the next section.

\section{Phase slips}

As the frequency difference $\Delta \omega$ inside the core region becomes stronger, the wavenumber of generated waves increases and, eventually, some pattern instabilities develop. These instabilities are accompanied by the appearance of phase slips and the formation of amplitude defects, and thus they cannot be described in the framework of the phase dynamics approximation given by Eq. (7). Phase slips accompany desynchronization phenomena and are closely related to the Eckhaus instability of plane waves [24].

Plane waves in the CGLE become unstable with respect to the Eckhaus instability if their wavenumber exceeds the threshold given by $k_{\mathrm{EI}}^{2}=(1+\alpha \beta) /$ $\left(3+\alpha \beta+2 \alpha^{2}\right)$. Two different types of spatiotemporal behavior are possible. The Eckhaus instability can be supercritical and modulated amplitude waves are stable solutions [25]. Alternatively, this instability may be subcritical and 
the perturbation does not saturate. Then, the wave is compressed until locally the amplitude drops down to zero at some time moments corresponding to phase singularities. Such events are associated with a phase slip of $2 \pi$, the disappearance of one wave and a subsequent readjustment of the wavenumber on both sides of the defect [24]. Also, in two space dimensions, spiral breakup into turbulence is then possible, a situation intensively discussed at the moment [26-28]. In this context, the distinction between convective and absolute Eckhaus instability is important [29]. If an infinitesimal perturbation of an unstable traveling wave state is advected away with the wave and asymptotically decays at any fixed position, the system is in the regime of convective Eckhaus instability. If this perturbation grows even at fixed positions, then the instability is called absolute. No matter what flavor of the Eckhaus instability is realized, the pacemaker represents a finite perturbation for the emitted wave. This perturbation grows down the flow and its effects are expected at a finite distance from the origin of the perturbation.

In the numerical simulations for the oscillatory medium with positive dispersion (Fig. 3), the initial condition represented a target pattern emitting stable waves near the Eckhaus instability. Then, the frequency difference $\Delta \omega$ in the core region (which is displayed in the bottom part of Fig. 3) was increased. After the time the perturbation needed to grow and modify the traveling wave, the amplitude drops down to zero (seen as the first black dot in Fig. 3b) and the phase slips. Then, a sequence of four phase slips occurs, with their respective locations moving at an approximately constant velocity towards the core. This motion is terminated at a certain distance from the core boundary. Eventually, a steady wave pattern with periodically generated phase slips is established. Note that the wavenumber in the far region becomes reduced and lies below the Eckhaus threshold ( $c f$. [24]). Thus, the waves emitted by a pacemaker undergo phase slips and a wave pattern with a short wavelength near the core and a long wavelength in the far region is established. Since the change of the frequency shift inside of the core and hence the size of the perturbation of the traveling wave state is of a finite size, the instability might still be convective or even absolute in line with the papers mentioned above. A detailed analysis would be necessary to clarify this question.

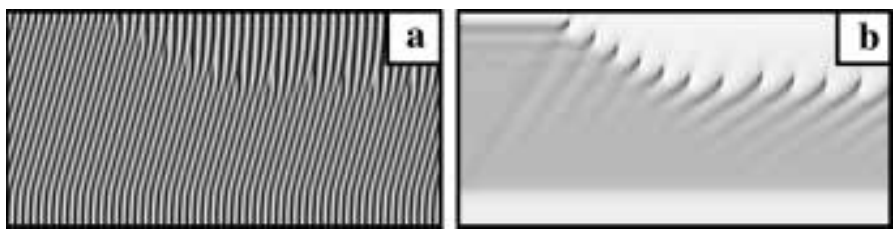

Fig. 3. Onset of the Eckhaus instability and formation of phase slips in a target pattern. Space-time diagrams of (a) $\operatorname{Re} A$ and (b) $|A|$ are shown in grey scale; $\Delta \omega=0.207$, $\Omega=0.55, \alpha=0.55, \beta=1.0, R=14.8, T=500, L=160$ (only half of the system is displayed, with the core in the lower part of the figure). 


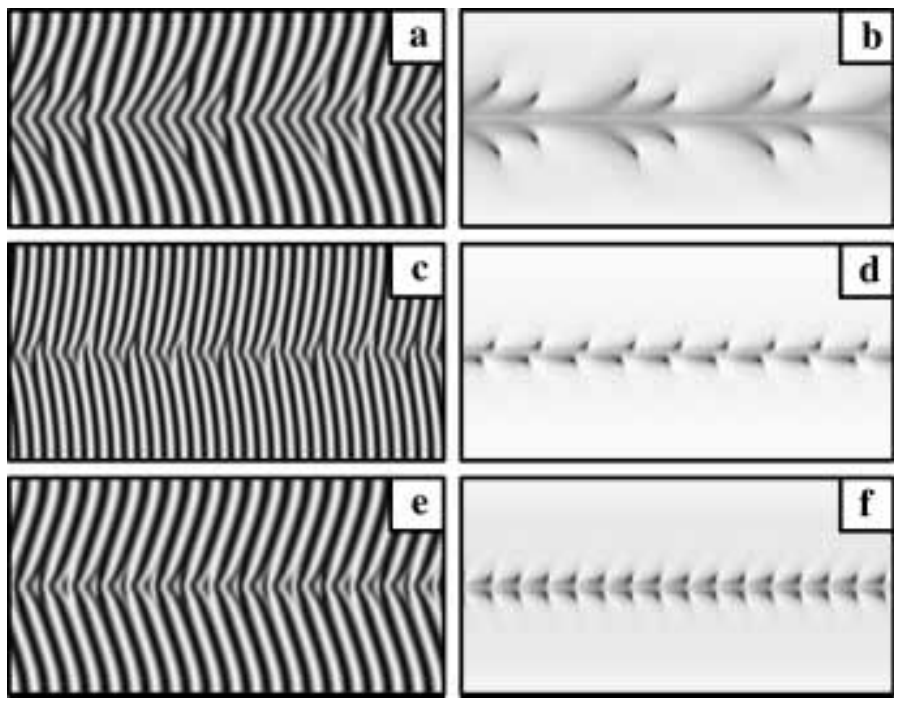

Fig. 4. Space-time diagrams of different wave patterns exhibiting phase slips: (a,b) $\Omega=$ $0.5, \Delta \omega=0.6, \alpha=0.5$; (c,d) $\Omega=0.8, \Delta \omega=0.7, \alpha=0.8$; (e,f) $\Omega=0.5, \Delta \omega=0.8, \alpha=$ 0.5 . Other parameters are $\beta=1.0, R=2.0, T=250, L=80$. The left row displays $\operatorname{Re} A$, the right row shows $|A|$ in grey scale.

When the frequency $\Delta \omega$ in the core region is further increased, the location where phase slips take place moves closer to the core boundary and more complex wave patterns become possible (Fig. 4). Phase slips may arrange in groups which are then repeated (Fig. 4b). For a larger $\Delta \omega$ (Fig. 4c,d), the phase slips on the two sides of the core do not occur simultaneously, giving rise to an asymmetric breakdown of waves. At a still larger value of the frequency difference $\Delta \omega$ (Fig. 4e,f), phase slips again occur symmetrically but very close to the core boundary. Note that because the local wavenumber is strongly changing near the boundary, the formation of phase slips in this case cannot be simply interpreted as a result of an Eckhaus instability for plane waves but rather as a desynchronization phenomenon. Generally, for increasing $\Delta \omega$ the location where phase slips occur moves closer to the core until the core boundary is reached. Further increase of the frequency shift leads to an increase of the frequency of the phase slips. The frequency of the waves in the far region depends strongly on the parameter $\alpha$ and its mismatch with $\Delta \omega$, as illustrated in Fig. 5.

The simulations displayed in Figs. 4 and 5 are examples where phase slips occur for a wave source. When the local oscillation frequency is decreased inside the core in a medium with positive dispersion, a wave sink is instead formed (see Fig. 1b). If the local decrease $\Delta \omega$ of the oscillation frequency is strong, phase slips can develop in the wave sink pattern. This is shown in Fig. 6. We see that the effective oscillation frequency of the pattern is now different 

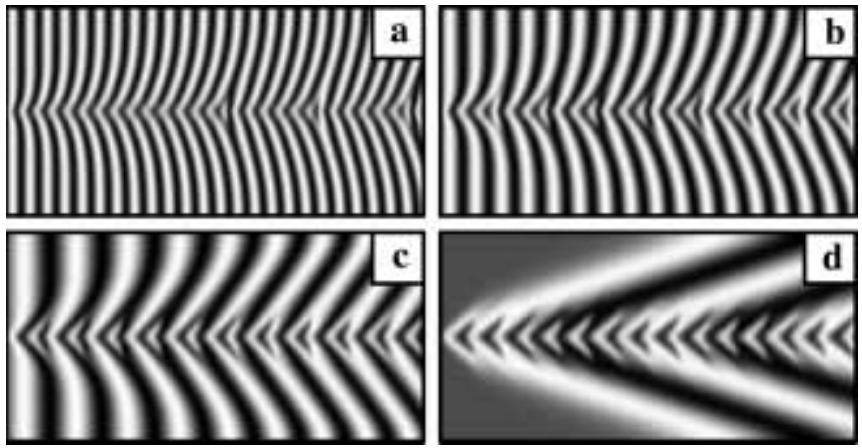

Fig. 5. Evolution of target patterns with phase slips under decrease of the parameter $\alpha$ : (a) $\alpha=0.75$; (b) $\alpha=0.5$; (c) $\alpha=0.25$; (d) $\alpha=0.0$. Other parameters are $\Delta \omega=0.7$, $\omega=0, \beta=1.0, R=2.0, T=200, L=80$.

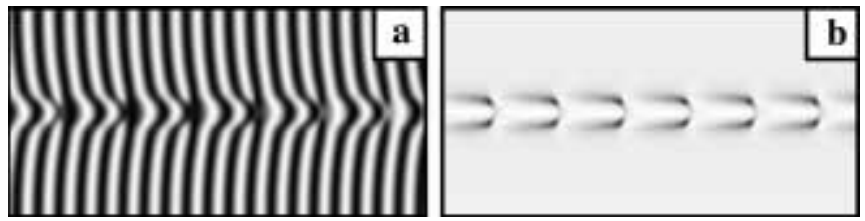

Fig. 6. Phase slips in a wave sink in a medium with positive dispersion; $\Delta \omega=0.3, \Omega=$ $0.5, \alpha=0.5, \beta=1.0, R=5.2, T=250, L=80$.
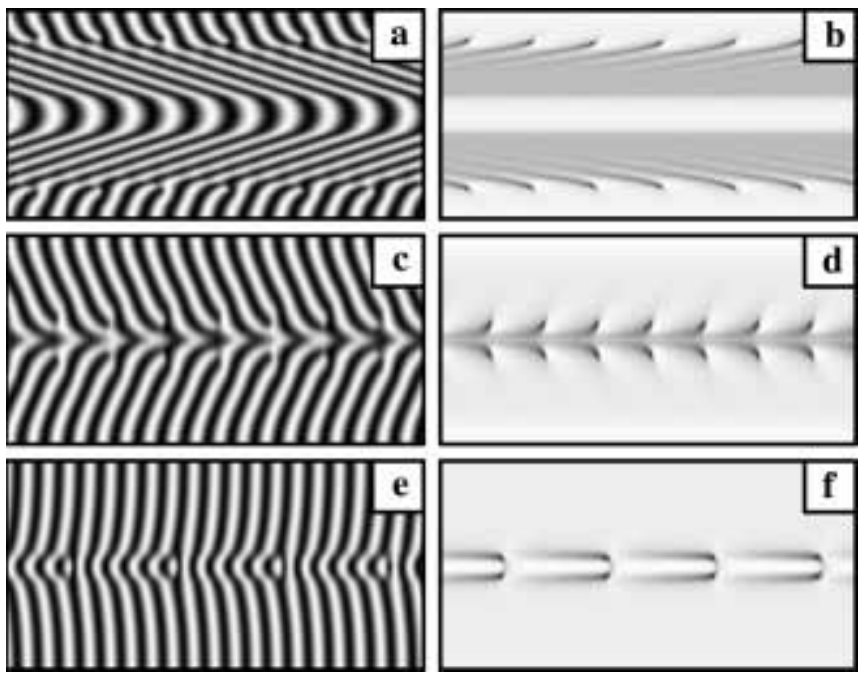

Fig. 7. Wave patterns with phase slips in media with negative dispersion: (a,b) $\Delta \omega=$ $0.2072, R=14.8, L=160$; (c,d) $\Delta \omega=0.65, R=2.0, L=80$; (e,f) $\Delta \omega=0.3, R=4$, $L=80$. Other parameters are: $\Omega=0.45, \alpha=-0.55, \beta=-1.0, T=250$. 
from the frequency of uniform oscillation outside of the core. Some oscillations in the outside region are unable to induce waves propagating inside the core. Here, the phase slips are bound to the border of the core.

In media with negative dispersion, phase slips are also possible (Fig. 7). In Fig. 7a,b we show a target pattern with inward propagating, Eckhaus-unstable waves $(\Delta \omega<0)$. Phase slips occur here at a finite distance from the core boundary, before the waves have actually reached it. Fig. 7c,d displays another example of an inward propagating target pattern where the decrease $\Delta \omega$ of the local frequency inside the core is stronger and the phase slips already appear near the core boundary. Finally, Fig. 7e,f shows phase slips in the wave pattern corresponding to a wave sink with $\Delta \omega>0$.

\section{Discussion}

Our study provides evidence that complex wave patterns with a rich variety of spatiotemporal behaviours can be produced in oscillatory media by introducing a heterogeneity, i.e., a core region where the local oscillation frequency is modified. Depending on the parameters of the medium, which determine the dispersion of waves, target patterns with outward and inward propagating waves can be observed. Moreover, localized sink patterns are also possible.

When the difference of the oscillation frequency in the core region is increased, wave regimes with periodic formation of phase slips are observed. In these regimes, the effective oscillation frequency inside the core becomes different from the frequency of oscillations in the far region. The phase slips occur because the medium is no longer able to compensate the frequency shift by the propagation of waves. Hence, desynchronization takes place and oscillations in the core region become decoupled from the rest of the medium. In a different setting, this effect has previously been discussed by Sakaguchi [30] who has used mode truncation to analyze the onset of desynchronization in the CGLE.

Though our analysis has been performed for a model system described by the CGLE, its results would probably remain qualitatively correct for other oscillatory media with anharmonical oscillations. In experiments with the photosensitive Belousov-Zhabotinsky reaction, the oscillation frequency can easily be controlled by changing the local light intensity. In this way, heterogeneities with different shapes, sizes and strengths can be created (see [17]). Varying these parameters and reaction conditions, it should be possible to create different pacemakers and observe the onset of desynchronization in the system.

\section{Acknowledgement}

One of the authors (M.S.) is very grateful to L. Brusch for stimulating discussions and useful remarks on the Eckhaus instability. 


\section{References}

1. F. W. Schneider and A. F. Münster, Nichtlineare Dynamik in der Chemie, SpektrumVerlag, Heidelberg (1996).

2. Chemical Waves and Patterns (R. Kapral, K. Showalter, Eds.) Kluwer Academic Publishers, Dordrecht (1995).

3. A. N. Zaikin and A. M. Zhabotinsky, Nature 255 (1970) 535.

4. S. Nasuno, M. Sano and Y. Sawada, J. Phys. Soc. Jpn. 58 (1989) 1875.

5. S. Jakubith, H. H. Rotermund, W. Engel, A. v. Oertzen and G. Ertl, Phys. Rev. Lett. 65 (1990) 3013.

6. M. Assenheimer and V. Steinberg, Phys. Rev. Lett. 70 (1993) 3888.

7. K. J. Lee, Phys. Rev. Lett. 79 (1997) 2907.

8. J. Christoph et al., J. Chem. Phys. 110 (1999) 8614.

9. H. Sakaguchi, Prog. Theor. Phys. 87 (1992) 241.

10. A. B. Rovinsky, A. M. Zhabotinsky and I. R. Epstein, Phys. Rev. E 56 (1997) 2412.

11. M. Stich, M. Ipsen and A. S. Mikhailov, Phys. Rev. Lett. 86 (2001) 4406.

12. C. Vidal and A. Pagola, J. Phys. Chem. 93 (1989) 2711.

13. K. I. Agladze and V. I. Krinsky, in Self-Organization, Autowaves and Structures far from Equilibrium (V. I. Krinsky, Ed.) Springer, Berlin (1984) pp. 147-149.

14. J. J. Tyson and P. C. Fife, J. Chem. Phys. 73 (1980) 2224.

15. H. Nagashima, J. Phys. Soc. Jpn. 60 (1991) 2797.

16. A. E. Bugrim, M. Dolnik, A. M. Zhabotinsky and I. R. Epstein, J. Phys. Chem. 100 (1996) 19017.

17. H. Mahara, T. Saito, Y. Amagishi, H. Nagashima and T. Yamaguchi, J. Phys. Soc. Jpn. 69 (2000) 3552.

18. Y. Kuramoto, Chemical Oscillations, Waves, and Turbulence, Springer-Verlag, Berlin (1984).

19. S. Alonso, I. Sendiña-Nadal, V. Pérez-Muñuzuri, J. M. Sancho and F. Sagués, Phys. Rev. Lett. 87 (2001) 078302.

20. I. A. Aranson and L. Kramer, cond-mat/0106115 (2001).

21. M. Ipsen, F. Hynne and P. G. Sørensen, Chaos 8 (1998) 834.

22. A. S. Mikhailov, Foundations of Synergetics I, Springer, Berlin (1994).

23. J. A. Sepulchre, G. Dewel and A. Babloyantz, Phys. Lett. A 147 (1990) 380.

24. B. Janiaud, A. Pumir, D. Bensimon, V. Croquette, H. Richter and L. Kramer, Physica D 55 (1992) 269.

25. L. Brusch, M. G. Zimmermann, M. van Hecke, M. Bar and A. Torcini, Phys. Rev. Lett. 85 (2000) 86.

26. S. M. Tobias and E. Knobloch, Phys. Rev. Lett. 80 (1998) 4811.

27. M. Bär and M. Or-Guil, Phys. Rev. Lett. 82 (1999) 1160.

28. B. Sandstede and A. Scheel, Phys. Rev. E 62 (2000) 7708.

29. I. S. Aranson, L. Aranson, L. Kramer and A. Weber, Phys. Rev. A. 46 (1992) R2992.

30. H. Sakaguchi, Prog. Theor. Phys. 80 (1988) 743. 